\title{
Neurochemistry of Enamoramiento
}

\author{
Gabriel Miranda Nava* \\ Neurologist and Clinical Neurophysiologist, Mexico

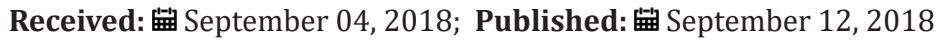 \\ *Corresponding author: Gabriel Miranda Nava, Neurologist and Clinical Neurophysiologist, Mexico
}

\section{Opinion}

Are you from people who live stormy romances? Did you find it difficult to separate yourself from a partner when in the back you intuit that it does not do you good? Did you know that the dynamics of love could be explained by neurotransmitters? It is increasingly known the fact of the process of falling in love through the chemicals that run through the brain and allow the communication of neurons with each other and through the human body, to be able to put together a word that can explain the whole context: thought. The first phase of an infatuation has the bases in the social standards with respect to a concept, the beauty, that distances of a single form depending on the time, the social classes, the fashions and the diverse ethnic groups that live in our world; in some groups obesity can be attractive, in other groups, more western tend to thin the body, in others less like a type of makeup, in others some deformity in the body, usually female, to attract male, to feel wanted and wanted; here it is important to note the presence of substances that enhance this attraction, pheromones, which through the nostrils we can detect and which are amplified through the use of a soap or a perfume (agreeing that a perfume or lotion is it smells different between one being and another) and even many years will pass and that smell will linger in our memories. When we see the person, we see it, we feel it, we even hear it, we can immediately fall in love, and here the key substance is called phenylethylamine, which is secreted by the limbic system, and causes total shock, fortunately for a short time, perhaps seconds, a few seconds, because then it would be tragic for the organism, since the organs of the senses and the autonomic nervous system, responsible for respiration, heart rate or intestinal transit, are altered; it is believed that this biochemical reaction has its analog in the toloache and the tummy water, but that instead of causing an effect of falling in love, they cause intoxication and damage to the central nervous system. Subsequently, the human body must wash this substance from the human body through other neurotransmitters, called vasoactive amines, such as dopamine and norepinephrine, but they are not harmless and cause some "havoc" in our body: have you felt that your chest is beating? Do you feel the famous butterflies in your stomach? Do you have many sighs? Is it hard for you to fall asleep? Well, you have to thank these substances, here is the process of falling in love or "what are you nailing".

Later you must feel a calm, to be in peace completely, calmly, as we would say "in the clouds" and without pain to anything, it is as Tin-tan would say ". I feel straight languid", but again it is an effect of the so-called endorphins, which are substances that simulate opium, so we are involved in a natural drug. This series of steps become more powerful and intense through sexual relationships; if these appear in early stages may appear a disenchantment, but at the passage of this cascade sex plays a role of brutal intensifier of the couple that is even the trigger of many crimes of passion and intrafamily violence, due to secreted estrogens and testosterone, which is where it allows human beings to "mark their territory". One can fall into excessive attachments in each stage, so we cannot explain how that attractive woman walks with a man who treats her badly or who does not even possess a physical attractiveness, or viscerally, because it is very well explained by the fact of creating a "Addiction" to each of the substances in the respective stage. As we said love, like many other human feelings, have to do with secretion of certain substances in the brain, called neurotransmitters, which have the function of regulating behavior and emotions and that will be reflected in our actions. But not only in emotions can the role of these neurotransmitters be explained, as it can be seen in the daily movement (a dance, walking, moving an arm) or is reflected in our thinking, mathematical or subjective as in the appreciation of a work of art, or in the reactions of stress to a danger and how the substances in question act for any action of our days, or the logical response to depression or manic-depressive disorder. Apparently, every time we give a logical explanation of the emotions, we want to find a justification, a cause causae, an irreversible determinism to follow a behavior, and that is not true, we cannot blame our way of thinking on the biochemical reactions They are always impregnated in our consciousness, they can always be modified by ourselves with totally volitional behaviors. Am I not the person responsible for making the decision to determine this relationship so damaging to me? Not at all, the best and only way to face these and other 
situations is through free will, to face and take responsibility for our decisions, since the effect of these neurotransmitters modulates the answers, but does not make us mentally deficient people that prevent take the reins of our way, it does not go like this, free will or that is the same being responsible for ourselves is the only answer, because in the end, it does not stop being the daughter of our neurochemical responses.
(C) (i) This work is licensed under Creative

To Submit Your Article Click Here: Submit Article

DOI: 10.32474/SJPBS.2018.01.000111

\begin{tabular}{|l|l|}
\hline SJPBS & $\begin{array}{c}\text { Scholarly Journal of Psychology } \\
\text { and Behavioral Sciences }\end{array}$ \\
Assets of Publishing with us
\end{tabular}

